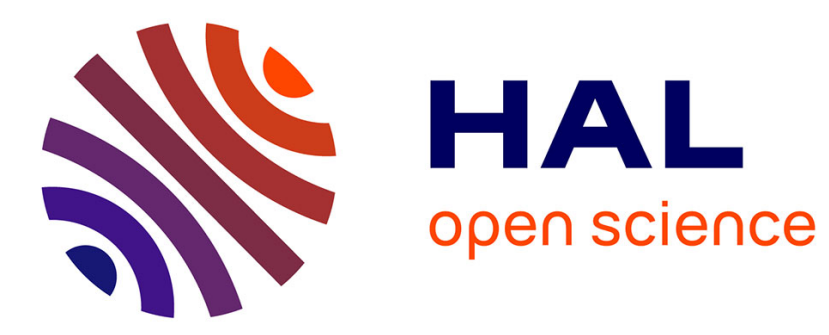

\title{
New fiber laser architecture with transform-limited nonlinear spectral compression
}

\author{
Sonia Boscolo, S.K. Turitsyn, Christophe Finot
}

\section{To cite this version:}

Sonia Boscolo, S.K. Turitsyn, Christophe Finot. New fiber laser architecture with transform-limited nonlinear spectral compression. 14th International Conference on Transparent Optical Networks, Jul 2012, Coventry, United Kingdom. pp.We.C1.6. hal-00731490

\section{HAL Id: hal-00731490 \\ https://hal.science/hal-00731490}

Submitted on 12 Sep 2012

HAL is a multi-disciplinary open access archive for the deposit and dissemination of scientific research documents, whether they are published or not. The documents may come from teaching and research institutions in France or abroad, or from public or private research centers.
L'archive ouverte pluridisciplinaire HAL, est destinée au dépôt et à la diffusion de documents scientifiques de niveau recherche, publiés ou non, émanant des établissements d'enseignement et de recherche français ou étrangers, des laboratoires publics ou privés. 


\title{
New Fiber Laser Architecture with Transform-Limited Nonlinear Spectral Compression
}

\author{
Sonia Boscolo ${ }^{1}$, Sergei K. Turitsyn ${ }^{1}$ and Christophe Finot ${ }^{2, *}$ \\ ${ }^{1}$ Institute of Photonic Technologies, Aston University, Birmingham, B47ET, United Kingdom \\ ${ }^{2}$ Laboratoire Interdisciplinaire Carnot de Bourgogne, UMR 6303, BP 47870, 21078 Dijon Cedex, France \\ * corresponding author : christophe.finot@u-bourgogne.fr
}

\begin{abstract}
We numerically demonstrate a new fiber laser architecture supporting spectral compression of negatively chirped pulses in passive normally dispersive fiber. Such a process is beneficial for improving the energy efficiency of the cavity as it prevents narrow spectral filtering from being highly dissipative. The proposed laser design provides an elegant way of generating transform-limited picosecond pulses.
\end{abstract}

Keywords: fiber lasers, self-similar propagation, nonlinear spectral compression.

\section{INTRODUCTION}

Mode-locked fiber laser technologies are fast growing solutions to provide stable, compact and cost-effective picosecond and sub-picosecond pulse sources. The non-trivial interplay between the effects of gain, dispersion and nonlinearity in a fiber laser cavity leads to a variety of possible pulse shaping mechanisms not yet fully explored. Short-pulse fiber lasers based on soliton formation in anomalous-dispersion cavities [1] , dispersionmanaged solitons in cavities with a dispersion map, and dissipative solitons in all-normal-dispersion cavities [2] have been demonstrated. Self-similar evolution of parabolic pulses, so-called "similaritons", in the passive fiber of a laser has also been observed, and has led to major performance increases in pulse energy over previouslystudied evolutions [3]. Recently, self-similar evolution in the gain segment of a fiber laser was demonstrated in a laser with an anomalous-dispersion segment [4], in a Raman fiber laser [5] and in an all-normal dispersion fiber laser [6]. In the latter configuration a narrow-band spectral filter provides the dominant mechanism for seeding the self-similar evolution in the amplifier by compensating both the broad pulse duration and bandwidth after the gain segment $[6,7]$.

In this paper, we propose a new fiber laser design that takes advantage of the process of spectral compression arising from nonlinear pulse propagation in optical fiber [8]. We numerically demonstrate than both highly chirped similariton pulses and transform-limited picosecond pulses can be generated with the proposed scheme. Nonlinear spectral compression yields increased spectral brightness compared to direct spectral filtering.

\section{LASER CAVITY ARCHITECTURE AND NUMERICAL MODEL}

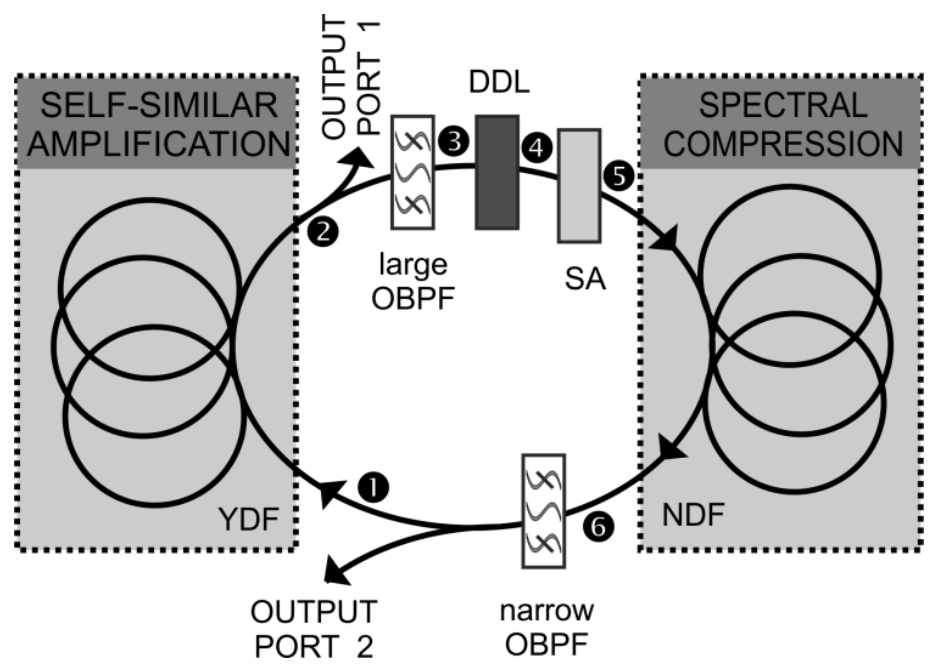

Figure 1. Laser configuration. YDF: Ytterbium doped fiber, OBPF: optical bandpass filter, DDL: dispersive delay line, SA: saturable absorber, NDF: normally dispersive fiber.

We consider a loop fiber laser configuration as shown in Fig. 1. A 4-m-long segment of ytterbium-doped fiber (YDF) provides pulse amplification and supports similariton dynamics with nonlinear attraction. A large optical bandpass filter (OBPF) is placed after the fiber amplifier to enhance stability of the laser operation. After the filter, the pulse enters a dispersive delay line (DDL; realizable experimentally with a pair of diffraction gratings or an hollow core fiber) which provides anomalous group-velocity dispersion (GVD) with negligible nonlinearity, and imparts a negative frequency chirp onto the positively (normally) chirped pulse as produced by 
the fiber amplifier. The DDL is followed by a saturable absorber (SA) element, and a 3.3-m-long segment of single-mode fiber with normal GVD, which provides nonlinear spectral compression of the negatively chirped, large-bandwidth input pulse. Indeed, for the negatively chirped pulse entering the fiber, where the long and the short wavelengths are in the trailing and leading edges, respectively, the effect of self-phase modulation is to redistribute both the long and the short wavelengths toward the center wavelength and therefore to spectrally compress the pulse instead of spectrally broadening it [8-10]. This results in significant increase of the energy spectral density. Such spectral compression is the key feature of our laser set-up. A narrow OBPF is used after the fiber to suppress the spectral wings due to imperfect compression. Two output couplers (OCs) are placed after the gain fiber and after the second OBPF, respectively.

Numerical simulations are based on a modified nonlinear Schrödinger equation,

$$
\frac{\partial \psi}{\partial z}=-i \frac{\beta_{2}}{2} \frac{\partial^{2} \psi}{\partial t^{2}}+i \gamma|\psi|^{2} \psi+\frac{1}{2} g * \psi
$$

Here, $\psi(z, t)$ is the slowly varying envelope of the pulse in a co-moving frame, $\beta_{2}=23 \mathrm{fs} / \mathrm{m}$ and $\gamma=4.410^{-3}$ $/ \mathrm{W} / \mathrm{m}$ are the respective GVD and Kerr nonlinearity parameters of the fiber, * represents the Fourier convolution, and $g(t)$ is the inverse Fourier transform of the gain spectrum given by

$$
g=\frac{g_{0}}{1+W / W_{0}+\left(\omega-\omega_{0}\right)^{2} / \Omega_{g}^{2}} \approx \frac{g_{0}}{1+W / W_{0}}\left[1-\left(\frac{\omega-\omega_{0}}{\Omega_{g}}\right)^{2}\right]
$$

where $g_{0}=30 \mathrm{~dB} / \mathrm{m}$ is the small-signal gain (non-zero only for the gain fiber), $\omega_{0}$ is the central angular frequency, $\Omega_{g}$ is the gain bandwidth, which is chosen to correspond to $8 \mathrm{THz}$ full-width at half-maximum (FWHM) bandwidth, $W(z)=\int \mathrm{d} t|\psi|^{2}$ is the pulse energy, and $W_{0}=600 \mathrm{pJ}$ is an effective gain saturation energy. The OBPFs are Gaussian in shape, specified by $h(\omega)=\exp \left[-\omega^{2} /\left(2 \Omega_{f}^{2}\right)\right]$, where $\Omega_{f}$ is the filter bandwidth which corresponds to $4 \mathrm{THz}$ and $0.25 \mathrm{THz}$ FWHM bandwidth for the first and second OBPF, respectively. The SA is modeled by a transfer function that describes its transmittance, $T(t)=1-q_{0} /\left[1+P(t) / P_{0}\right]$, where $q_{0}=0.8$ is the unsaturated loss, $P(z, t)=|\psi(z, t)|^{2}$ is the instantaneous pulse power, and $P_{0}=200 \mathrm{~W}$ is the saturation power. The DDL provides a total dispersion of $-197 \mathrm{fs}^{2}$. The OCs are modeled by a transfer function of the form: $\psi_{\text {pass }}(t) / \psi_{\text {in }}(t)=\sqrt{ } R$, where $R=0.4$ for the self-similar port (port 1 ), and $R=0.35$ for the spectral compression port (port 2). The linear losses inside the cavity are accounted for by $R$ without loss of generality. The numerical model is solved with the standard split-step Fourier method and the initial field is white noise. The simulations are run until stable operation of the laser is obtained after a finite number of round-trips.

\section{NONLINEAR PULSE SHAPING PROCESSES}

The steady-state pulse solution at different locations in the cavity is shown in Figs. 2 and 3. A typical feature of amplifier similaritons is that the pulses evolve toward a parabolic asymptotic solution [11,12]: the pulse is nearly parabolic in shape at the end of the gain fiber (Fig. 2a). The associated spectrum exhibits some structure, as expected for a parabola with finite (though large) chirp (Fig. 2b). This pulse has a time-bandwidth product (TBP) of approximately 32. Cancellation of the rather highly linear chirp outside the cavity can lead to high-quality temporally compressed pulses with durations as short as $100 \mathrm{fs}$ and peak powers well above $10 \mathrm{~kW}$. We can also see from Fig. 2 that both the temporal and spectral pulse widths at the end of the gain fibre are significantly larger than those at the fibre entrance. This is another signature of self-similar propagation [11,12] and modelocking, and is due to self-similar evolution in the gain fiber.
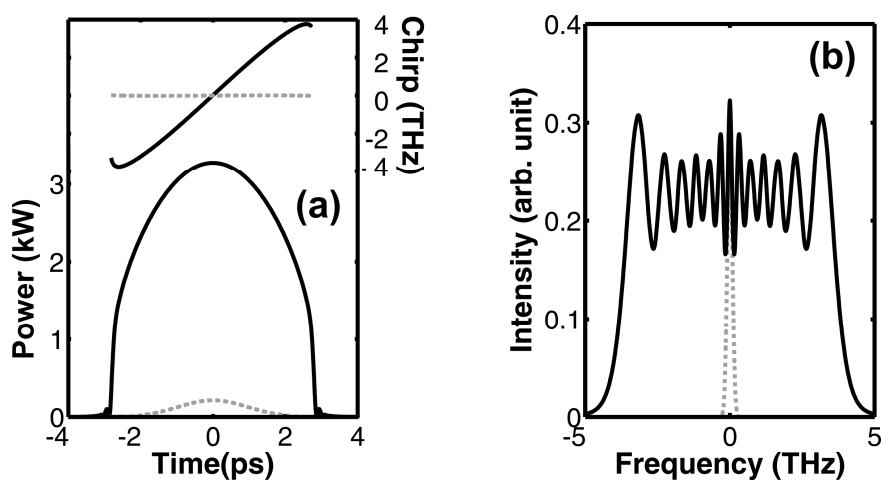

Figure 2. (a) Temporal intensity and chirp profiles, and (b) spectral intensity profiles of the pulse at the entrance ( location 1 in Fig. 1; dotted curves) and the exit (location 2 in Fig. 1; solid curves) of the gain fiber. 
Strong spectral narrowing of the pulse occurs upon nonlinear propagation in the passive normally dispersive fiber segment (Fig. 3b), leading to a compression factor (defined as the ratio of the input and output FWHM widths) of approximately 10 with an output FWHM width of $0.33 \mathrm{THz}$. The latter is comparable to the bandwidth of the narrow OBPF. The energy spectral density of the pulse is therefore largely increased, and nonlinear spectral compression prevents the narrow spectral filter from being highly dissipative, which is a drawback of previously reported configurations [6] where direct filtering introduces high energy losses. The output pulse from the fiber exhibits a nearly transform-limited picosecond profile with a TBP nearly equalizing the transform limit for a Gaussian pulse (0.441). Note that the generation of transform-limited picosecond pulses is very difficult to achieve in conventional laser cavities supporting self-similar evolution in normally dispersive fiber.
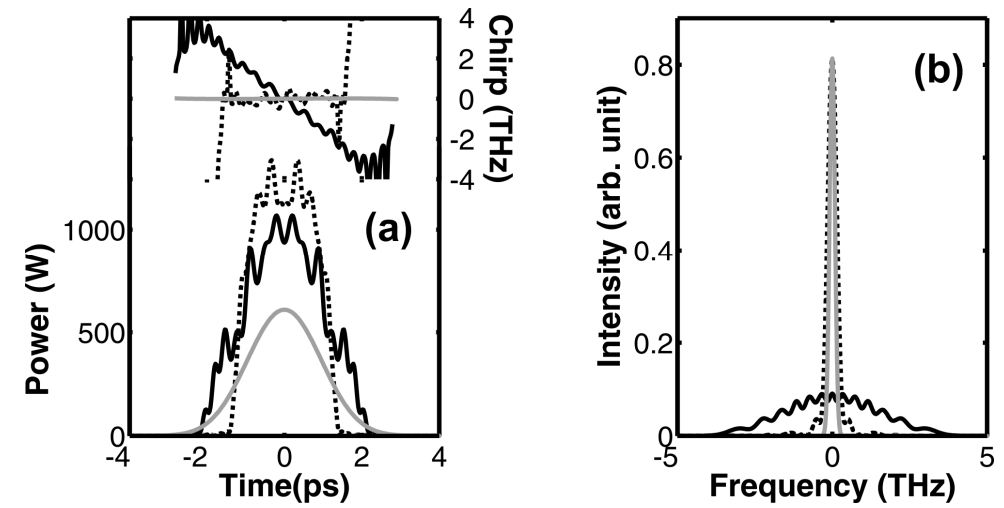

Figure 3. (a) Temporal intensity and chirp profiles, and (b) spectral intensity profiles of the pulse at the entrance of the passive fiber (location $\mathbf{5}$ in Fig. 1; solid black curves), the end of the passive fiber (location $\mathbf{6}$ in Fig. 1; dotted black curves), and after the narrow spectral filter (location $\mathbf{(}$ in Fig.1; solid gray curves).

The intra-cavity pulse dynamics is illustrated by plots of the evolution of the root-mean-square (rms) pulse duration and spectral bandwidth over one round-trip (Fig. 4). Two distinctly different nonlinear pulse shaping processes co-exist within the cavity: self-similar evolution in the gain fiber segment with monotonic temporal and spectral broadening of the pulse, and spectral compression in the passive fiber segment leading to large spectral narrowing. The first OBPF carves the output spectrum from the amplifier, and the linear DDL only affects the temporal width of the pulse.

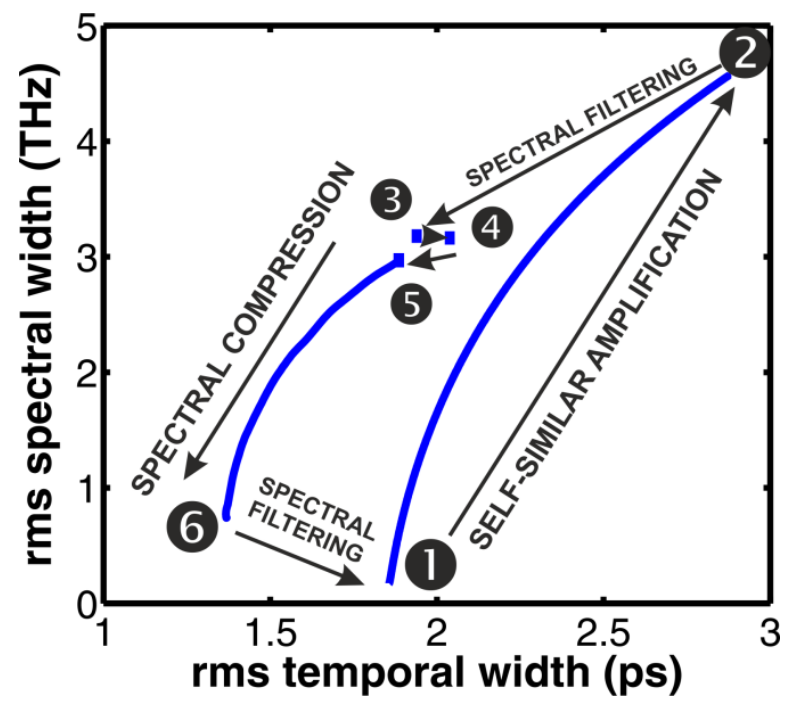

Figure 4. Phase diagram representing the combined evolution of the temporal and spectral RMS widths of the pulse over one round-trip. The locations in the cavity indicated in Fig. 1 are shown. 


\section{CONCLUSIONS}

We have presented a new fiber laser architecture that takes advantage of the spectral compression effect arising from nonlinear propagation of negatively chirped pulses in normally dispersive fiber to generate nearly transform-limited picosecond pulses. Through numerical modelling, we have shown that nonlinear spectral compression is beneficial for improving the energy efficiency of the cavity as it prevents narrow spectral filtering from being highly dissipative. Higher output pulse energies can be obtained by further scaling of the cavity parameters.

\section{ACKNOWLEDGEMENTS}

The authors would like to acknowledge support from the Engineering and Physical Sciences Research Council (EPSRC) (UK) (grant EP/FO2956X/1), the British Council (Alliance grant 10.002), the Région Bourgogne (PARI PHOTCOM), and the Ministère des Affaires étrangères et européenne (Alliance grant 22836ZJ).

\section{REFERENCES}

[1] F. M. Mitschke and L. F. Mollenauer, "Ultrashort pulses from the soliton laser," Opt. Lett., vol. 12, pp. 407 - 409, 6/1987 1987.

[2] F. W. Wise, et al., "High-energy femtosecond fiber lasers based on pulse propagation at normal dispersion," Laser \& Photonics Rev., vol. 2, pp. 58-73, 2/2008 2008.

[3] F. Ö. Ilday, et al., "Self-similar evolution of parabolic pulses in a laser," Phys. Rev. Lett., vol. 92, 213902(4), 2004.

[4] B. Oktem, et al., "Soliton-similariton fibre laser," Nat. Photonics, vol. 4, pp. 307-311, 5/2010 2010.

[5] C. Aguergaray, et al., "Experimental realization of a mode-locked parabolic Raman fiber oscillator," Opt. Express, vol. 18, pp. 8680-8687, 2010.

[6] W. H. Renninger, et al., "Self-similar pulse evolution in an all-normal-dispersion laser," Phys. Rev. A, vol. $82,2010$.

[7] B. G. Bale and S. Wabnitz, "Strong spectral filtering for a mode-locked similariton fiber laser," Opt. Lett., vol. 35, pp. 2466-2468, 2010.

[8] S. A. Planas, et al., "Spectral narrowing in the propagation of chirped pulses in single-mode fibers," Opt. Lett., vol. 18, pp. 699-701, 5/1993 1993.

[9] N. L. Markaryan, et al., "Spectral compression of ultrashort laser pulses," Kvantovaya Elektronika, vol. 18, pp. 865-867, Jul 1991.

[10] E. R. Andresen, et al., "Transform-limited spectral compression by self-phase modulation of amplitude shaped pulses with negative chirp," Opt. Lett., vol. 36, pp. 707-709, 2011.

[11] M. E. Fermann, et al., "Self-similar propagation and amplification of parabolic pulses in optical fibers," Phys. Rev. Lett., vol. 84, pp. 6010-6013, 6/2000 2000.

[12] C. Finot, et al., "Optical parabolic pulse generation and applications," IEEE J. Quantum Electron., vol. 45, pp. 1482-1489, 2009. 